\title{
Quantitative Model to Determine Permeability for Columnar Dendritic Structures
}

\author{
Yukinobu NATSUME, ${ }^{1) *}$ Daiki TAKAHASHI ${ }^{2)}$ Kasumi KAWASHIMA,${ }^{3)}$ Eiji TANIGAWA ${ }^{3)}$ and Kenichi OHSASA ${ }^{1)}$ \\ 1) Department of Materials Engineering, Akita University, Akita, 010-8502 Japan. \\ 2) Materials Research Laboratory, Kobe Steel Ltd., Kobe, 651-2271 Japan. \\ 3) Business Solution Development Group, Kobelco Systems Co., Kobe, 651-2271 Japan.
}

(Received on December 18, 2012; accepted on February 14, 2013)

\begin{abstract}
A new model that can quantitatively evaluate the permeability for columnar dendritic structures was developed by modifying the Kozeny constant in Kozeny-Carman's equation. The modified Kozeny constant consists of two terms: one accounting for the flow direction for primary arms of columnar dendrites and the other accounting for the tortuosity of channels in the dendritic structures. The permeability calculated by this new model was compared with that obtained in our previous simulations [Y. Natsume et al.: Tetsuto-Hagané, 99 (2013), 117] and from experiments other researchers [K. Murakami et al.: Acta metall., 31 (1983), 1417, 32 (1984), 1423, Liu et al.: Mater. Sci. Tech., 5 (1989), 1148] and the values were found to be in fairly good agreement with the compared values. In addition, we investigated the obtained quantitative model to determine permeability for use in computational studies of macrosegregation. To evaluate the permeability quantitatively using Kozeny-Carman's equation, the value of the specific surface area for dendrites is required. We introduced an assumption that the inverse of the specific surface area for columnar dendrites is proportional to the secondary arm spacing. By using this assumption in our modified model, the permeability can be determined using only the dendrite arm spacing and liquid volume fraction.
\end{abstract}

KEY WORDS: permeability; Kozeny-Carman model; columnar dendrite; macrosegregation; solidification.

\section{Introduction}

In large cast iron production, convection within the mushy region plays an important role in the phenomenon of macrosegregation. Macrosegregation represents solute composition inhomogeneity at the macroscopic scale, and it can lead to the formation of gross compositional defects that greatly affect the quality of casting products. It is therefore important to understand, study and control macrosegregation.

Macrosegregation arises mainly from fluid flow and microscopic scale segregation during solidification, and it follows that macrosegregation is controlled simultaneously by fluid flow and solute diffusion. Thus, macrosegregation models should describe both phenomena. In most macrosegregation models, flows of interdendritic liquid within the mushy region have been considered using Darcy's law. The governing equation is given as follows:

$$
\frac{D \boldsymbol{u}}{D t}=-\frac{1}{\rho} \nabla P+v \nabla^{2} \boldsymbol{u}-\frac{v}{K} \boldsymbol{u}
$$

where $\boldsymbol{u}$ is the vector of flow, $t$ is time, $\rho$ is fluid density, $P$ is pressure, $v$ is kinematic viscosity, and $K$ is permeability. The third term on the right side implies that Darcy's flow is considered only within the mushy region. Among the terms

\footnotetext{
* Corresponding author: E-mail: natsume@gipc.akita-u.ac.jp
} DOI: http://dx.doi.org/10.2355/isijinternational.53.838 used, permeability is a very important parameter as it affects the flow velocity within the mushy region.

The permeability for equiaxed dendrites has been investigated experimentally by Piwonka et al., ${ }^{1)}$ Apelian et al., ${ }^{2)}$ Streat and Weinberg, ${ }^{3)}$ Poirier et al., ${ }^{4,5)}$ and Takahashi et al. ${ }^{6-8)}$ Further, the permeability for columnar dendrites has been investigated experimentally by Streat and Weinberg, ${ }^{3)}$ Murakami et al., ${ }^{910)}$ Liu et al., ${ }^{11)}$ Nasser-Raff et al., ${ }^{12)}$ and Poirier et al. ${ }^{13)}$ The majority of these studies, which experimentally measured the permeability, were carried out before $1990 .{ }^{1-14)}$ In more recent studies, numerical simulations have been employed to estimate the permeability for dendritic structures, ${ }^{15-24)}$ owing to the difficulty of carrying out the experiments.

Among the experimental studies on the permeability of dendritic structures, Murakami et al. ${ }^{9,10)}$ and Liu et al. ${ }^{11)}$ measured the permeability of the interdendritic space of columnar dendrites using borneol-paraffin organic binary alloys. In columnar dendrites, the permeability is affected by the direction of flow; the value of permeability for a flow parallel to the primary dendrites is different from that for a flow normal to the dendrite. Therefore, they measured the permeability for both parallel and normal flows using multiple regression analysis. The permeability for a flow parallel to and normal to the primary dendrites, $K_{P-M}$ and $K_{N-M}$, respectively, as provided by them, is given as follows: 


$$
\begin{aligned}
& K_{P-M}=6.2 \times 10^{-13} d_{1}^{2.2} d_{2}^{-1.7} g_{L}^{3.2} \quad\left(0.19 \leq g_{L} \leq 0.61\right) \ldots .(2) \\
& K_{N-M}=8.8 \times 10^{-19} d_{1}^{1.3} d_{2}^{2.4} g_{L}^{3.2} \quad\left(0.19 \leq g_{L} \leq 0.66\right) \ldots \ldots(3)
\end{aligned}
$$

The permeability in these empirical equations is given as a function of the primary dendrite arm spacing (PDAS), $d_{1}$, the secondary dendrite arm spacing (SDAS), $d_{2}$, and the liquid volume fraction, $g_{L}$.

Nasser-Raff et al. ${ }^{12)}$ also measured the permeability for columnar dendrites of a $\mathrm{Pb}-\mathrm{Sn}$ alloy. Poirier et al. ${ }^{13)}$ derived empirical equations to calculate permeability using multiple regression analysis, which were then verified with the experimental results obtained by them as well as other researchers. Ganesan et al. ${ }^{15)}$ and Bhat et al. ${ }^{16)}$ estimated the permeability for columnar dendritic structures using a twodimensional numerical flow simulation. Poirier and Heinrich $^{17-19)}$ evaluated the permeability for columnar dendritic structures on the basis of the results from their experiments and simulations. According to them, the permeability for a flow parallel to and normal to the primary arms, $K_{P-P H}$ and $K_{N-P H}$, respectively, is given as follows:

$$
K_{P-P H}=\left\{\begin{array}{l}
3.75 \times 10^{-4} g_{L}^{2} d_{1}^{2} \quad\left(g_{L} \leq 0.65\right) \\
2.05 \times 10^{-7}\left[\frac{g_{L}}{1-g_{L}}\right]^{10.739} d_{1}^{2} \quad\left(0.65<g_{L} \leq 0.75\right) \\
0.074\left[\ln \left(1-g_{L}\right)^{-1}-1.49+2\left(1-g_{L}\right)-0.5\left(1-g_{L}\right)^{2}\right] d_{1}^{2}
\end{array}\right.
$$$$
\left(0.75<g_{L} \leq 1.0\right)
$$

$$
K_{N-P H}=\left\{\begin{array}{l}
1.09 \times 10^{-3} g_{L}^{3.32} d_{1}^{2} \quad\left(g_{L} \leq 0.65\right) \\
4.04 \times 10^{-6}\left[\frac{g_{L}}{1-g_{L}}\right]^{6.7336} d_{1}^{2} \quad\left(0.65<g_{L} \leq 0.75\right) \\
{\left[\begin{array}{l}
\left.-6.49 \times 10^{-2}+5.43 \times 10^{-2}\left(\frac{g_{L}}{1-g_{L}}\right)^{0.25}\right] d_{1}^{2}
\end{array}\right.}
\end{array}\right.
$$$$
\left(0.75<g_{L} \leq 1.0\right)
$$

The permeability in these equations is given as a function of the PDAS, $d_{1}$, and the liquid volume fraction, $g_{L}$. Poirier and Heinrich formulated the permeability in the case of liquid volume fractions less than 0.65 by experimental data using multiple regression analysis and that in the case of liquid volume fractions over 0.75 by numerical flow calculations. For liquid volume fractions between 0.65 and 0.75 , they introduced a relationship in order to best fit the two different equations. This is due to the fact that for liquid volume fractions around 0.7 , the values of permeability obtained from experiment varied greatly from those obtained from simulations. This difference was caused by their use of two-dimensional simulations. Therefore, the relationship between the permeability and the liquid volume fraction must be determined in three dimensions.

Recently, Brown et al. ${ }^{20)}$ carried out flow calculations in three dimensions to determine the permeability for equiaxed dendritic structures. The dendritic structures used in the flow calculations were simulated by a cellar automaton method. The permeability numerically determined was compared with that obtained from experimental data for an aluminum alloy. At low volume fractions, there was a reasonable correlation between the permeability obtained from the model and experimental data for equiaxed structures. Bernard and co-workers ${ }^{21,22)}$ used synchrotron X-ray microtomography in order to obtain the three-dimensional columnar dendritic structures of an Al-Cu alloy. They also calculated the permeability using flow calculations. There was a reasonable agreement between the calculated and experimentally determined permeability. However, this technique has an upper limit in reconstructed volume on the order of $1 \times$ $1 \times 0.6 \mathrm{~mm}$, and this domain of flow calculation will not be sufficiently large. As a result, the permeability determined by the flow calculation was compared with the experimental data for liquid volume fractions from 0.2 to 0.3 . In order to obtain a larger calculation domain, Madison et al. ${ }^{23)}$ obtained columnar dendritic structures by means of scanning the sample using an automated Robo-Met.3D serial sectioning system. The sample volume obtained with this technique was $2.3 \times 2.3 \times 1.5 \mathrm{~mm}$. They compared the permeability obtained from simulations with the experimental data from various researchers, and concluded that their simulated permeability was in general agreement with the permeability reported by various researchers employing either or both experimental and numerical approaches. However, in the approaches using the three-dimensional experimental structures, it still remains difficult to obtain the dendritic structures for higher liquid volume fractions.

In our previous work, ${ }^{24)}$ we created three-dimensional columnar dendritic structures by means of computer aided design (CAD) and carried out flow calculations in three dimensions. From the results of the flow calculation, we obtained the permeability of columnar dendrites for relatively higher liquid volume fractions. The simulated permeability was compared with the experimental data obtained by Murakami et al., ${ }^{9-11)}$ which showed that the calculated values were in excellent agreement with the experimental permeability for both parallel and normal flow directions. We also compared our results with those obtained from the empirical equations proposed by Murakami et al., shown in Eqs. (2) and (3) (Murakami's equations), and by Poirier et al., shown in Eqs. (4) and (5) (Poirier-Heinrich's equations); however, our simulated permeability was not in agreement with the permeability obtained from these equations. Thus, it is difficult to evaluate quantitatively the permeability of columnar dendritic structures using these empirical equations. A quantitative model to evaluate the permeability for columnar dendritic structures must therefore be developed.

Kozeny-Carman model or Kozeny-Carman's equation is well-known as one such quantitative model that can evaluate permeability for various porous media. The permeability calculated using Kozeny-Carman model, $K_{K C}$, is usually used for isotropic porous media and is described as follows:

$$
K_{K C}=\frac{1}{k S_{V}^{2}} \frac{g_{L}^{3}}{\left(1-g_{L}\right)^{2}}
$$

where $k$ is a constant and $S_{V}$ is surface area per unit volume for porous media. In general, $k$ is termed the "Kozeny constant" and is equal to five, which was obtained from exper- 
iments on grains of sand carried out by Carman. ${ }^{25)} S_{V}$ becomes $6 / d$ on assuming an isotropic porous media for spherical grains of average diameter $d$. Thus, the KozenyCarman model can be rewritten as follows:

$$
K_{K C}=\frac{d^{2}}{180} \frac{g_{L}^{3}}{\left(1-g_{L}\right)^{2}}
$$

Kozeny-Carman model can be used to determine the permeability of equiaxed dendritic structures as well, assuming that $d$ is the equiaxed grain size. However, for columnar dendritic structures, Eq. (7) cannot be used directly because the flow direction for columnar dendritic structures has not been considered in the original Kozeny-Carman model. Thus, in order for the Kozeny-Carman model to be used as a model for columnar dendritic structures, the equation must be modied.

In the present work, our purpose is to develop a new model that will be able to evaluate quantitatively the permeability for columnar dendritic structures. The new model is based on the Kozeny-Carman model. We modified the Kozeny constant, $k$, in Eq. (6) so as to consider the flow direction of interdendritic liquid for columnar dendritic structures; the modified Kozeny constant is termed $k_{m}$ in this paper. The model obtained by substituting $k$ in the original Kozeny-Carman's equation with $k_{m}$ is called the modified Kozeny-Carman model (MKC model). In order to examine the validity of the MKC model, the permeability obtained using the model was compared with the simulated permeability obtained in our previous work ${ }^{24)}$ and the experimental data reported in earlier works. ${ }^{9-14)}$ Furthermore, we evaluate the applicability of this quantitative model for numerical simulation of macrosegregation.

\section{Permeability from Three-Dimensional Flow Calcula- tions}

In our previous work, ${ }^{24)}$ we modeled three-dimensional columnar dendritic structures using CAD on the basis of the two-dimensional dendritic morphologies calculated by phase-field simulation. We then carried out the flow calculations in three dimensions for the two types of flow directions (parallel and normal), as shown in Fig. 1. In the flow calculations, the superficial velocity, $u_{S}$, and the pressure loss, $\Delta P$, were calculated and then substituted into Darcy's equation to calculate the permeability for columnar dendritic

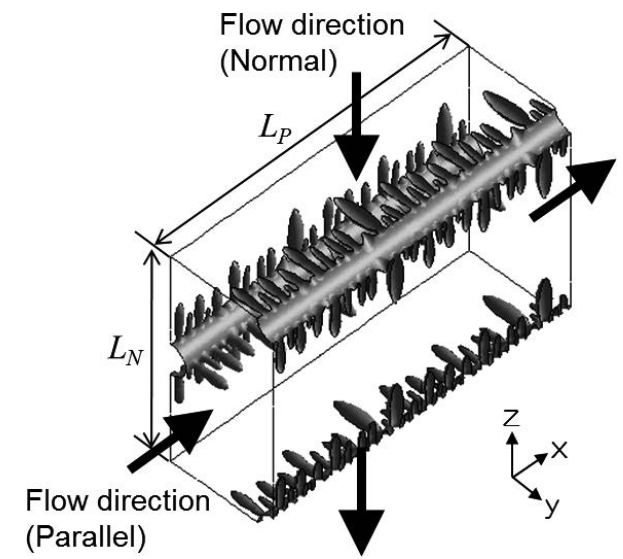

Fig. 1. Conditions of flow directions used in the three-dimensional flow calculations. structures. Darcy's equation is given as follows:

$$
K=\frac{\mu g_{L} u_{S} L}{\Delta P}
$$

where $\mu$ is viscosity and $L=L_{P}$ and $L=L_{N}$, for flows parallel to and normal to the primary arms, respectively. $L_{P}$ and $L_{N}$ are shown in Fig. 1. Figure 2 shows the six types of columnar dendritic structures modeled in our previous work, with liquid volume fractions of $0.56,0.66,0.70,0.77,0.87$, and 0.95 . PDAS is the distance between the primary arms of closest approach, and it was $320 \mu \mathrm{m}$ in the previous work. In the present work, we also carried out flow calculations for columnar dendritic structures having PDASs of $100 \mu \mathrm{m}$ and $420 \mu \mathrm{m}$. Table 1 shows the simulated permeability for flows parallel to and normal to the primary arms having PDASs of $320 \mu \mathrm{m}$ (our previous work), $100 \mu \mathrm{m}$ and $420 \mu \mathrm{m}$ (our present work); the values of SDAS, $d_{2}$, and the inverse of the solid-liquid interfacial area per unit volume, $1 / S_{V}$, are also provided in Table 1 . The simulated permeability increased with increasing PDAS for columnar dendritic structures having the same volume fraction and increased with increasing liquid volume fractions for structures having the same PDAS. Also to be noted is that the simulated permeability for a flow parallel to the primary arms was several times larger than that for a flow normal to the primary arms.

\section{Modified Kozeny-Carman Model (MKC Model)}

\subsection{Influence of Flow Directions}

As mentioned in section 1, the original Kozeny-Carman model (Eq. (6)) gives the permeability for isotropic porous media. This model consists of a Kozeny function related to
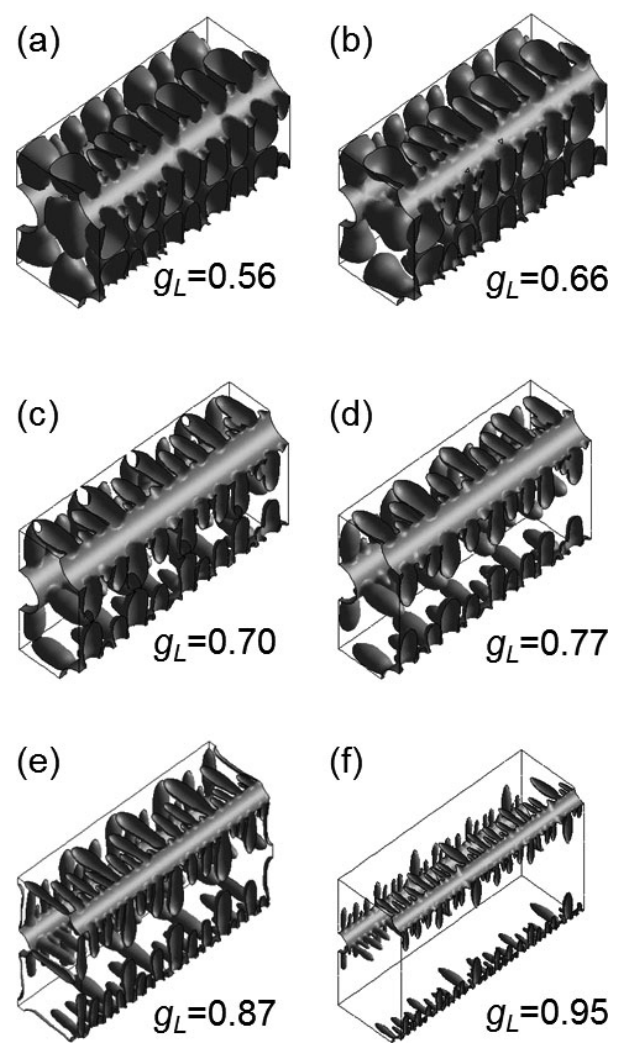

Fig. 2. The three-dimensional columnar dendritic structures modeled using CAD. 
Table 1. Values of the liquid volume fraction, $g_{L}$, the primary dendrite arm spacing, $d_{1}$, the secondary dendrite arm spacing, $d_{2}$, and the inverse of surface area per volume, $1 / S_{V}$, obtained from the three-dimensional columnar dendritic structures, and the permeability obtained from our simulations.

\begin{tabular}{ccccccc}
\hline $\begin{array}{c}\text { No. } \\
\text { (Fig. 2) }\end{array}$ & $g_{L}$ & $\begin{array}{c}\mathrm{d}_{1} \\
(\mu \mathrm{m})\end{array}$ & $\begin{array}{c}\mathrm{d}_{2} \\
(\mu \mathrm{m})\end{array}$ & $\begin{array}{c}1 / S_{V} \\
(\mathrm{~m})\end{array}$ & $\begin{array}{c}K_{P} \\
\left(\mathrm{~m}^{2}\right)\end{array}$ & $\begin{array}{c}K_{N} \\
\left(\mathrm{~m}^{2}\right)\end{array}$ \\
\hline (a) & 0.56 & 100 & 25.4 & $6.52 \times 10^{-6}$ & $1.06 \times 10^{-11}$ & $5.55 \times 10^{-12}$ \\
(b) & 0.66 & 100 & 25.4 & $5.76 \times 10^{-6}$ & $3.61 \times 10^{-11}$ & $9.12 \times 10^{-12}$ \\
(c) & 0.70 & 100 & 24.9 & $5.61 \times 10^{-6}$ & $5.99 \times 10^{-11}$ & $1.29 \times 10^{-11}$ \\
(d) & 0.77 & 100 & 22.3 & $5.56 \times 10^{-6}$ & $7.79 \times 10^{-11}$ & $2.75 \times 10^{-11}$ \\
(e) & 0.87 & 100 & 17.7 & $3.47 \times 10^{-6}$ & $1.10 \times 10^{-10}$ & $3.69 \times 10^{-11}$ \\
(f) & 0.95 & 100 & 11.5 & $2.77 \times 10^{-6}$ & $4.20 \times 10^{-10}$ & $2.24 \times 10^{-10}$ \\
& & & & & & \\
(a) & 0.56 & 320 & 81.3 & $2.09 \times 10^{-5}$ & $1.08 \times 10^{-10}$ & $5.68 \times 10^{-11}$ \\
(b) & 0.66 & 320 & 81.3 & $1.84 \times 10^{-5}$ & $3.70 \times 10^{-10}$ & $9.34 \times 10^{-11}$ \\
(c) & 0.70 & 320 & 79.7 & $1.82 \times 10^{-5}$ & $6.13 \times 10^{-10}$ & $1.32 \times 10^{-10}$ \\
(d) & 0.77 & 320 & 67.8 & $1.80 \times 10^{-5}$ & $7.97 \times 10^{-10}$ & $2.82 \times 10^{-10}$ \\
(e) & 0.87 & 320 & 56.6 & $1.12 \times 10^{-5}$ & $1.13 \times 10^{-9}$ & $3.77 \times 10^{-10}$ \\
(f) & 0.95 & 320 & 36.8 & $8.85 \times 10^{-6}$ & $4.30 \times 10^{-9}$ & $2.29 \times 10^{-9}$ \\
& & & & & & \\
(a) & 0.56 & 420 & 106.7 & $2.74 \times 10^{-5}$ & $1.87 \times 10^{-10}$ & $9.78 \times 10^{-11}$ \\
(b) & 0.66 & 420 & 106.7 & $2.42 \times 10^{-5}$ & $6.37 \times 10^{-10}$ & $1.61 \times 10^{-10}$ \\
(c) & 0.70 & 420 & 104.6 & $2.39 \times 10^{-5}$ & $1.06 \times 10^{-9}$ & $2.28 \times 10^{-10}$ \\
(d) & 0.77 & 420 & 89.0 & $2.36 \times 10^{-5}$ & $1.37 \times 10^{-9}$ & $4.85 \times 10^{-10}$ \\
(e) & 0.87 & 420 & 74.2 & $1.47 \times 10^{-5}$ & $1.94 \times 10^{-9}$ & $6.50 \times 10^{-10}$ \\
(f) & 0.95 & 420 & 48.3 & $1.16 \times 10^{-5}$ & $7.41 \times 10^{-9}$ & $3.94 \times 10^{-9}$ \\
\hline & & & & & &
\end{tabular}

the liquid volume fraction, $g_{L}{ }^{3} /\left(1-g_{L}\right)^{2}$, and a term that depends on the shape of the porous media, $1 / k S_{V}$. The Kozeny function represents the qualitative variation of permeability for liquid volume fractions from 0 to 1 . The term related to the shape of the media consists of two constants: the Kozeny constant, $k$, and the specific surface area, $S_{V}$. The values of $k$ and $S_{V}$ are used to determine the permeability quantitatively.

In the case of isotropic porous media such as grains of sand, the constants are generally defined as $k=5$ and $S_{V}=$ $6 / d$. On the other hand, in anisotropic porous media such as bundles of fibers, the value of $k$ is not a constant and assumes different values for different orientations of the exposed surface. Fowler and Hertel ${ }^{26)}$ investigated the relationship between the geometrical morphology of textile fiber and the Kozeny constant, $k$. In case no transverse pressure gradients can be sustained, they showed that $k$ is given as follows:

$$
k=k^{\prime}\left\langle\cos ^{2} \theta\right\rangle_{A V}
$$

where $k^{\prime}$ is the shape factor for flow in channels of various cross sections and $\theta$ is the angle between the direction of passage and the $\mathrm{x}$ axis. $\left\langle\cos ^{2} \theta>_{A V}\right.$ represents the average value of $\cos ^{2} \theta$. On the basis of the equation derived by Fowler and Hertel, Sullivan and $\mathrm{Hertel}^{27)}$ described $k$ without including the shape factor, $k^{\prime}$, as follows:

$$
k=\frac{3}{\left\langle\sin ^{2} \phi\right\rangle_{A V}}
$$

where $\phi$ is the angle between the direction of macroscopic flow and the normal to an element of surface exposed to the flow. They predicted that, for a bed of spheres, it should take a value of 4.5, while for a bed of cylinders parallel and normal to the flow direction, it should take values of 3 and 6 , respectively. Using experiments, they confirmed that these predictions are valid within the limits of experimental error. Assuming that a bundle of fibers corresponds approximately to the primary dendrite arms of columnar dendrites, Eq. (10) can be suitable for columnar dendritic structures. However, the influence of the secondary dendrite arms is not involved in Eq. (10). Thus, $k$ must be modified to account for a dendrite morphology with both primary and secondary arms.

\subsection{Tortuosity}

$k$ is generally given as $k=c \tau^{2}$, where $c$ is a constant and $\tau$ is a parameter known as the "tortuosity factor". This factor $\tau$ takes into account the fact that the interdendritic channels are neither straight nor smooth, and $\tau=1$ means straight or smooth channels and $\tau>1$ means tortuous channels. In dendritic structures, $\tau$ must reflect the dendrite morphology, and thus, it must be determined on the basis of microstructural information such as interdendritic channels, namely, PDAS and SDAS. However, it is difficult to theoretically derive the relationship between $\tau$ and this information, and hence, only few models to determine $\tau$ for dendritic structures are known. Recently, Santos and Melo ${ }^{28)}$ developed a model of the permeability for columnar dendritic structures on the basis of Hargen-Poiseuille law. They proposed a heuristic relationship to estimate the tortuosity for flows parallel to and normal to the primary arms. In the relationship, the tortuosity factor was related to $d_{1}, d_{2}$, and $g_{L}$ as follows:

$$
\tau=1+\sum_{m=0}^{n}\left(\frac{d_{2}}{d_{1}}\right)^{\left(g_{L}\right)^{m}}
$$

where $n$ and $m$ are arbitrary integers. The terms $d_{2} / d_{1}$ used in Eq. (11) is a good attempt to represent a feature of dendrite morphology, and in cases where the value of $d_{1}$ is fixed, the value of $\tau$ approaches one as the value of $d_{2}$ becomes small. Additionally, in the cellular structures, where $d_{2}=0, \tau$ is equal to one. This means straight or smooth channels. In general, since SDAS is smaller than PDAS, the value of $\tau$ will be grater than one. This is valid because the channels are not smooth and straight. By this argument, the equation proposed by Santos and Melo ${ }^{28)}$ to estimate $\tau$ for dendritic structures is reasonable. However, in Eq. (11), the tortuosity of the Kozeny-Carman model cannot be directly replaced because this equation is the best fit for a model of permeability based on the Hargen-Poiseuille law as follows:

$$
K=\frac{g_{L}^{2} A}{8 N \pi \tau^{3}}
$$

Here $A$ is the cross-sectional area and $N$ is the number of channels per unit area. Since $\tau$ to the power of 3 is used in Eq. (12), the value of $\tau$ found from Eq. (11) will be too small for the Kozeny constant, $k=c \tau^{2}$. Therefore, we propose a new heuristic relationship for the tortuosity factor, $\tau$, as follows:

$$
\tau=1+\sum_{m=1}^{n} m\left(\frac{d_{2}}{d_{1}}\right)^{m}
$$

This equation for $\tau$ also includes parameters $d_{1}, d_{2}$, and $g_{L}$. The optimal value of $\tau$ for the MKC model can be obtained 
using Eq. (13), which will be discussed in section 3.3.

\subsection{Modification of the Kozeny Constant}

In the present work, we propose MKC model with a modified Kozeny constant, $k_{m}$, based on the equation $k=c \tau^{2}$. Both the influence of flow directions (parallel and normal) for columnar dendritic structures and a new relationship of tortuosity have been considered in the modified model. First, to incorporate the influence of flow directions, we replaced the constant $c$ with the Kozeny constant proposed by Sullivan and Hertel (Eq. (10)). This means that the Kozeny constant is equal to Eq. (10) when $\tau=1$. As described in the previous section, $\tau=1$ describes the case with no secondary arms, i.e., a cellular structure with only primary arms, similar to the case of a bundle of fibers. Second, for the tortuosity, we used the new heuristic relationship given in Eq. (13). Substituting these two relationships into $k=c t^{2}$, the equations of the MKC model and $k_{m}$ becomes

$$
\begin{gathered}
K_{M K C}=\frac{1}{k_{m} S_{V}^{2}} \frac{g_{L}^{3}}{\left(1-g_{L}\right)^{2}} \ldots \ldots \ldots . . . . . . \\
k_{m}=\frac{3}{\left\langle\sin ^{2} \phi\right\rangle_{A V}}\left(1+\sum_{m=1}^{n} m\left(\frac{d_{2}}{d_{1}}\right)^{m}\right)^{2}
\end{gathered}
$$

The fitting parameter is only an integer $n$. On comparing the permeability obtained from the present model and that obtained in our previous work ${ }^{24)}$ and in works of others, ${ }^{10-12)}$ the best fit for flows parallel to and normal to the primary arms were obtained when $n=1$ and $n=2$, respectively. Hence, in the MKC model, the permeability can be determined using variables related to the dendritic structures, namely, $S_{V}, g_{L}, d_{1}$, and $d_{2}$. In general, however, the flow directions within the mushy region are not always classified as parallel and normal for primary arms; they tend to differ for different situations. From the definition of $\phi, \phi$ cannot exactly express the angle between the flow direction and the growth direction of the primary arms, except parallel and normal directions. Thus, an angle between the flow direction and the growth direction of the primary arms is defined as $\alpha$. Figure 3 shows a schematic illustration to explain the definition of the angle $\alpha$ between the flow direction and the growth direction of the primary arm. Assuming that the relationship between $\alpha$ and $k$ is linear, then $k_{m}(\alpha)$ is described

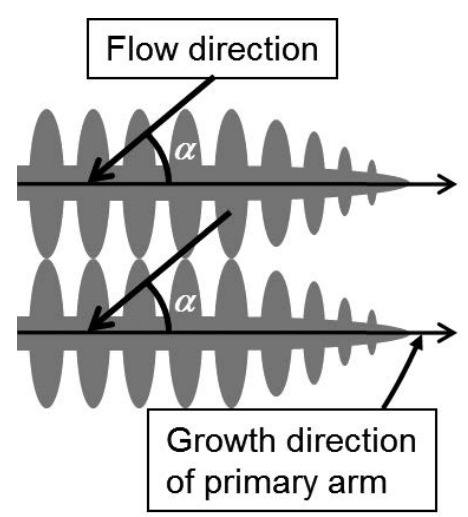

Fig. 3. A schematic illustration to explain the definition of the angle $\alpha$ between the flow direction and the growth direction of the primary arms. as follows:

$$
k_{m}(\alpha)=[3 f(\alpha)+6(1-f(\alpha))]\left(1+\sum_{m=1}^{n} m\left(\frac{d_{2}}{d_{1}}\right)^{m}\right)^{2} \ldots . .
$$

where $k=3$ and $k=6$ were used for flows parallel $(\alpha=0)$ to and normal ( $\alpha=\pi / 2)$ to the primary arms, respectively. $f$ $(\alpha)$ is a function of $\alpha$ that must satisfy the conditions $f(0)=$ 1 and $f(\pi / 2)=0$, for example, $f(\alpha)=\cos (\alpha), f(\alpha)=\cos ^{2}(\alpha)$, etc.

\section{Model Validation}

Validation of the MKC model was carried out by comparison with permeabilities obtained from three other methods, namely, (1) from three-dimensional flow calculations (section 2), (2) from experiments performed by Murakami et al., ${ }^{9-11)}$ and (3) from the empirical equations of Murakami et $a l .^{9-11)}$ and Poirier et al. ${ }^{17)}$

\subsection{Comparison with the Permeability from Three- Dimensional Flow Calculations}

In our previous work, we confirmed that the permeability obtained by three-dimensional flow calculations (our simulation) was in good agreement with the experimental data obtained by Murakami et al. ${ }^{9-11)}$ In this section, we compare the MKC model with the results of our previous simulation.

Figure 4 shows the relationship between the liquid volume fractions and the permeability in our previous simulation and the present MKC model at $d_{1}=320 \mu \mathrm{m}$. The per-
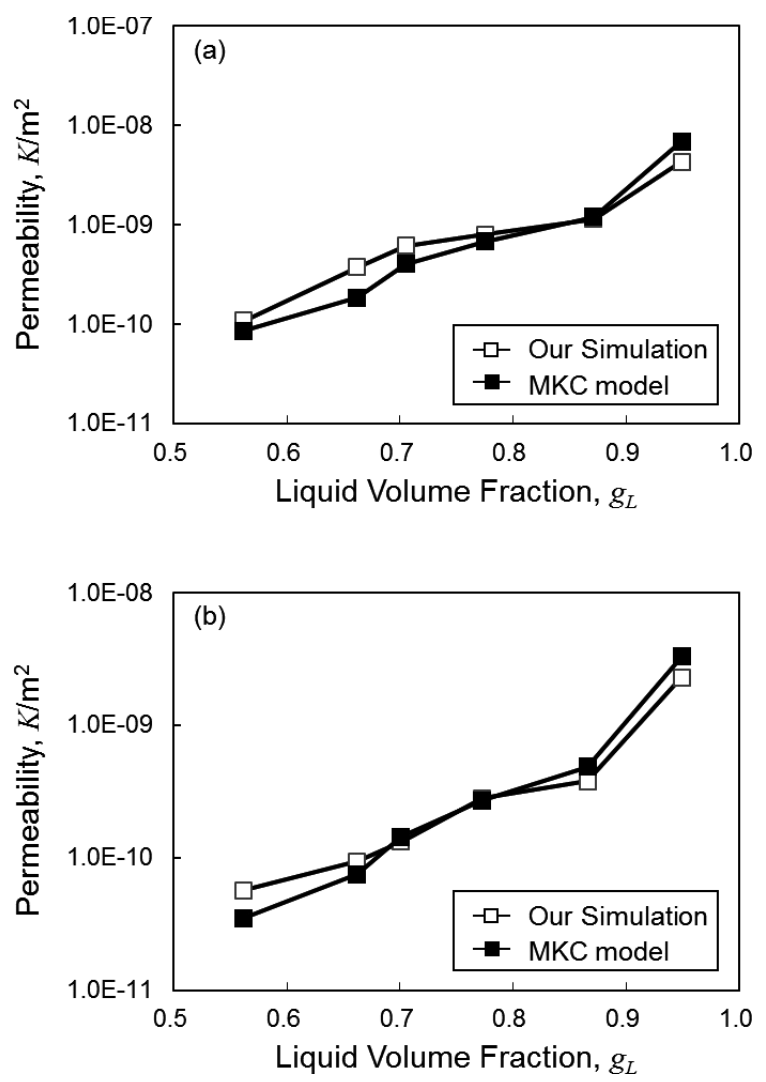

Fig. 4. Relationship between the liquid volume fractions and the permeability in our previous simulations and the present MKC model at $d_{1}=320 \mu \mathrm{m}$. (a) For a flow parallel to the primary arms. (b) For a flow normal to the primary arms. 
meability for flows parallel to and normal to the primary arms is shown in Figs. 4(a) and 4(b), respectively. The values of $S_{V}, g_{L}$, and $d_{2}$ used to calculate the permeability of the MKC model are the same as those used in our previous simulation, which are shown in Table 1 . The values of permeability obtained from the MKC model are in fairly good agreement with those from our previous simulation for both flow directions. From these results, it is confirmed that the MKC model is an effective and quantitative model to determine permeability for columnar dendritic structures.

\subsection{Comparison with Experimental Data}

In this section, we compare the permeability obtained from the MKC model with the experimental data obtained by Murakami et al. ${ }^{9,10)}$ and Liu et al. ${ }^{11)}$ They measured the permeability of borneol-paraffin organic binary alloys for flows parallel to and normal to the primary arms. In their experiments, the permeability of columnar dendritic structures for liquid volume fractions between 0.19 and 0.66 was investigated. Compared with other studies that experimentally measured the permeability, ${ }^{3,12,13)}$ their investigation included a wider range of volume fractions. Moreover, they used samples of columnar dendritic structures with different PDAS of $d_{1}=420$ and $320 \mu \mathrm{m}$. Hence, a lot of experimental data is available to validate the MKC model.

Figures 5 and $\mathbf{6}$ show the relationship between the liquid
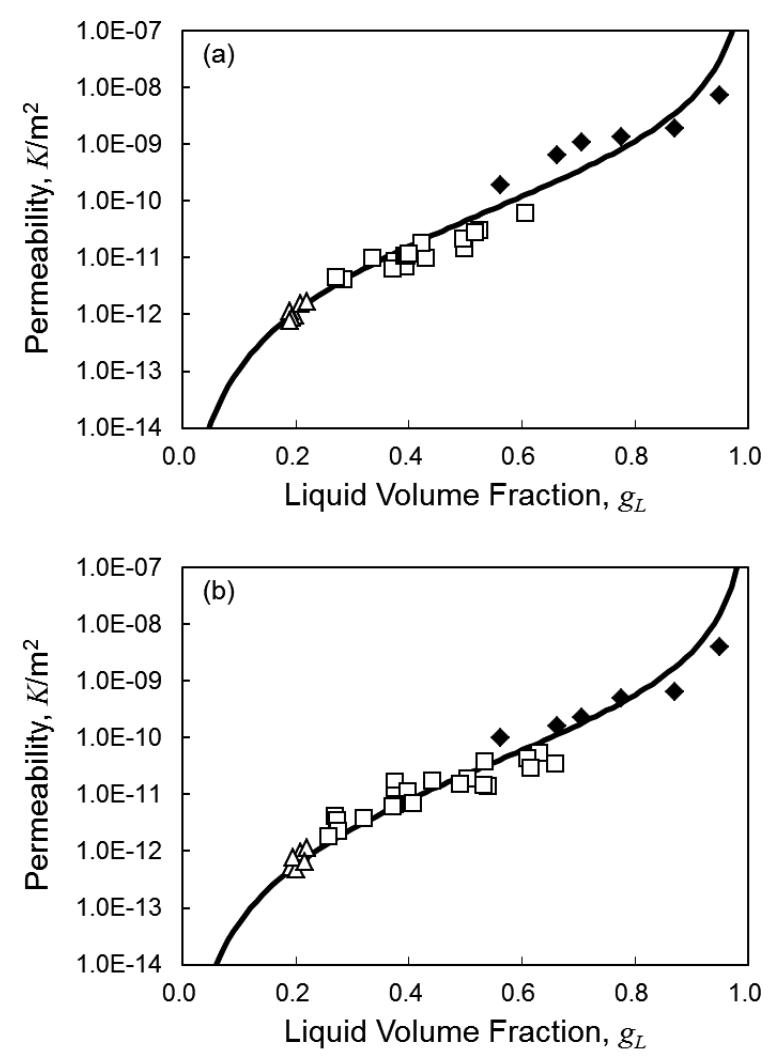

\begin{tabular}{rl|}
$\bullet$ & Our Simulation \\
$\square$ & Experiment: Murakami et al. ${ }^{9,10)}$ \\
$\Delta$ & Experiment: Liu et al. ${ }^{11)}$ \\
- & MKC model
\end{tabular}

Fig. 5. Relationship between the liquid volume fractions and the permeability obtained in the experiments ${ }^{9-11)}$ and the MKC model for a PDAS of $420 \mu \mathrm{m}$. (a) For a flow parallel to the primary arms. (b) For a flow normal to the primary arms. volume fractions and permeability obtained in the experiments for PDAS of $420 \mu \mathrm{m}$ and $320 \mu \mathrm{m}$. For comparison, the results of our simulation are also shown in these figures. In order to calculate the permeability using the MKC model, the values of $1 / S_{V}$ and $k_{m}$ for volume fractions from 0 to 1 are required. If the morphology and the size of the dendrite are known exactly, the values of $1 / S_{V}$ and $k_{m}$ can be evaluated. However, it is very difficult to know the morphology and size exactly for dendritic structures. Thus, we used the average values of $1 / S_{V}$ and $k_{m}$ for six columnar dendritic structures in our simulation. The values of $1 / S_{V}$ for each structure are shown in Table 1, while those of $k_{m}$ are shown in Table 2 for flows parallel to and normal to the primary arms for each volume fraction. The average values of $1 / S_{V}$

Table 2. Values of modified Kozeny constant, $k_{m}$, for each columnar dendritic structure.

\begin{tabular}{ccc}
\hline & \multicolumn{2}{c}{$k_{m}$} \\
$g_{L}$ & parallel & normal \\
\hline 0.56 & 4.72 & 11.48 \\
0.66 & 4.72 & 11.48 \\
0.70 & 4.68 & 11.31 \\
0.77 & 4.41 & 10.49 \\
0.87 & 4.15 & 9.21 \\
0.95 & 3.73 & 7.82 \\
\hline
\end{tabular}
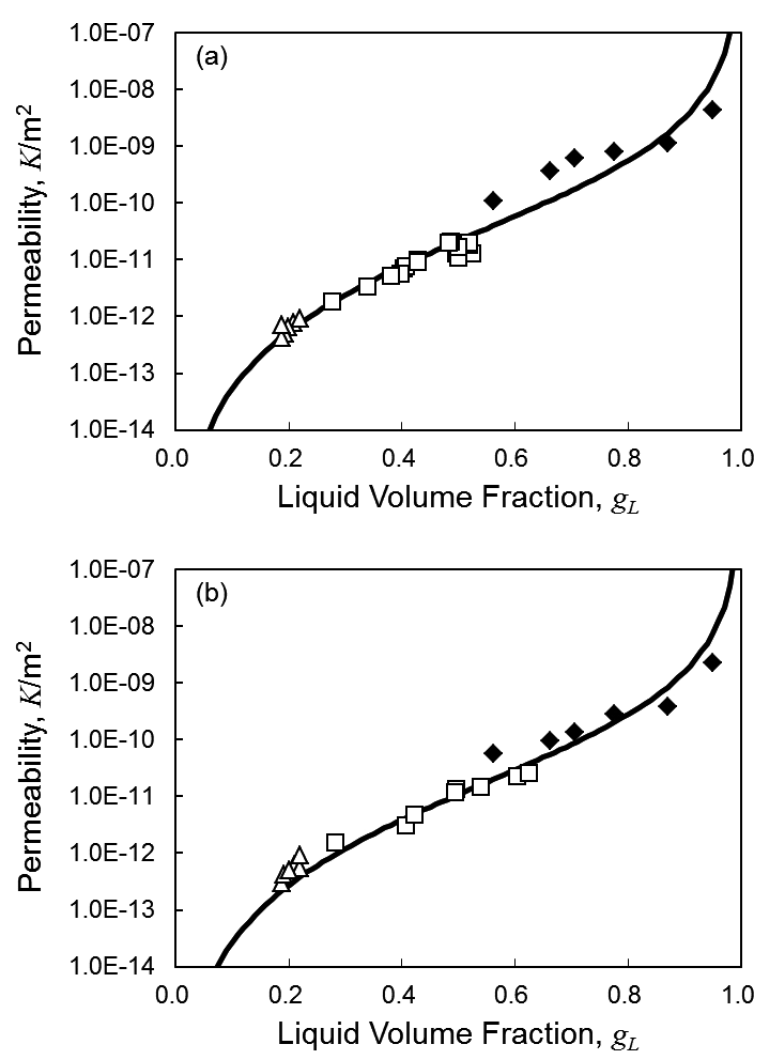

$\begin{array}{ll}- & \text { Our Simulation } \\ \square & \text { Experiment: Murakami et al. }{ }^{9,10)} \\ \Delta & \text { Experiment: Liu et al. }{ }^{11)} \\ - & \text { MKC model }\end{array}$

Fig. 6. Relationship between the liquid volume fractions and the permeability obtained in the experiments ${ }^{9-11)}$ and the MKC model for a PDAS of $320 \mu \mathrm{m}$. (a) For a flow parallel to the primary arms. (b) For a flow normal to the primary arms. 
are $2.16 \times 10^{-5} \mathrm{~m}$ and $1.65 \times 10^{-5} \mathrm{~m}$ for columnar dendritic structures having PDAS of $420 \mu \mathrm{m}$ and $320 \mu \mathrm{m}$, respectively. The average values of $k_{m}$ for flows parallel to and normal to the primary arms are 4.4 and 10.3 , respectively.

First, a comparison of the permeability calculated using the MKC model (see Table 1) under four conditions, two flow directions (parallel and normal) and two PDASs (420 $\mu \mathrm{m}$ and $320 \mu \mathrm{m}$ ), was done. The permeability for the flow parallel to the primary arms was larger than that for the flow normal to, and the permeability for the PDAS of $420 \mu \mathrm{m}$ was larger than that for the PDAS of $320 \mu \mathrm{m}$. Next, a comparison between the permeability calculated using the MKC model and that obtained from experiments and our simulation was done (see Figs. 5 and 6). The results of MKC model are in fairly good agreement with experiments and our simulation for all conditions. This also confirmed that the MKC model is valid as a model to evaluate quantitatively the permeability for columnar dendritic structures.

\subsection{Comparison with Empirical Equations}

As a final validation, we compared the results of our model with those obtained from the empirical equations postulated by Murakami et al. ${ }^{9-11)}$ (Murakami's equations: Eqs. (2) and (3)) and Poirier et al. ${ }^{17)}$ (Poirier-Heinrich's equations: Eqs. (4) and (5)).

Figure 7 shows the relationship between the liquid vol-
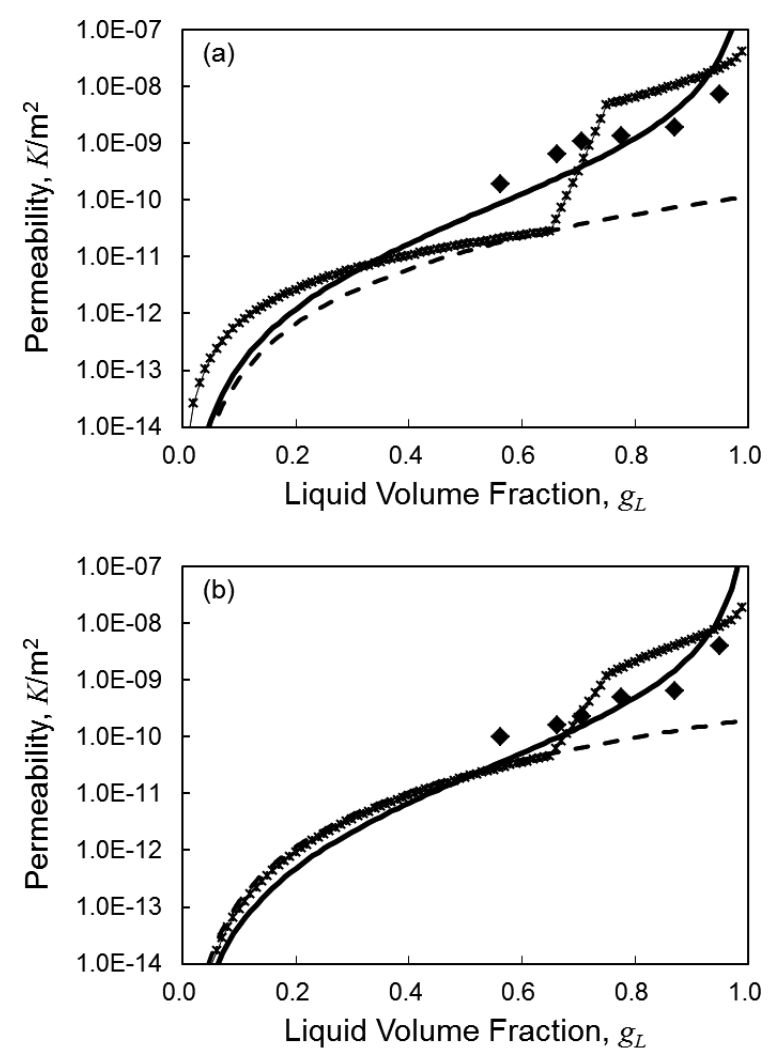

\begin{tabular}{ll|}
\hline - Our Simulation \\
- & MKC model \\
-- Murakami's equations ${ }^{9-11)}$ \\
$*$ Poirier-Heinrich's equations ${ }^{17)}$ \\
\hline
\end{tabular}

Fig. 7. Relationship between the liquid volume fractions and the permeability obtained using the empirical equations ${ }^{9-11,17)}$ and the MKC model for a PDAS of $420 \mu \mathrm{m}$ and an SDAS of $115 \mu \mathrm{m}$. (a) For a flow parallel to the primary arms. (b) For a flow normal to the primary arms. ume fractions and the permeability obtained using the empirical equations and the MKC model for a PDAS of 420 $\mu \mathrm{m}$ and an SDAS of $115 \mu \mathrm{m}$. The values of the PDAS and SDAS are as determined from the experiments of Murakami et al. ${ }^{9-11)}$ and the value of $1 / S_{V}$ used is $2.16 \times 10^{-5} \mathrm{~m}$. For comparison, the results of our simulation are also shown in these figures. First, on comparing the three curves of permeability for the flow parallel to the primary arms (Fig. 7(a)), we found that for lower liquid volume fractions of less than about 0.6 , the curve calculated using the MKC model is close to that from the Murakami's equations. However, the difference in permeability increases with increasing liquid volume fractions. On the other hand, although the curves obtained using the Poirier-Heinrich's equations and the MKC model cross at liquid volume fractions of around 0.35 , 0.7 , and 0.95 , the variation in permeability obtained from the Poirier-Heinrich's equations is close to that obtained using the MKC model for volume fractions of 0 to 1 . Next, on comparing the three curves of permeability for the flow normal to the primary arms (Fig. 7(b)), we found that the curves obtained using the MKC model are in fairly good agreement with those obtained using both empirical equations, except Murakami's equations for liquid volume fractions higher than 0.65. The curve obtained using Murakami's equations for higher liquid volume fractions is extrapolated owing to the lack of experimental data.
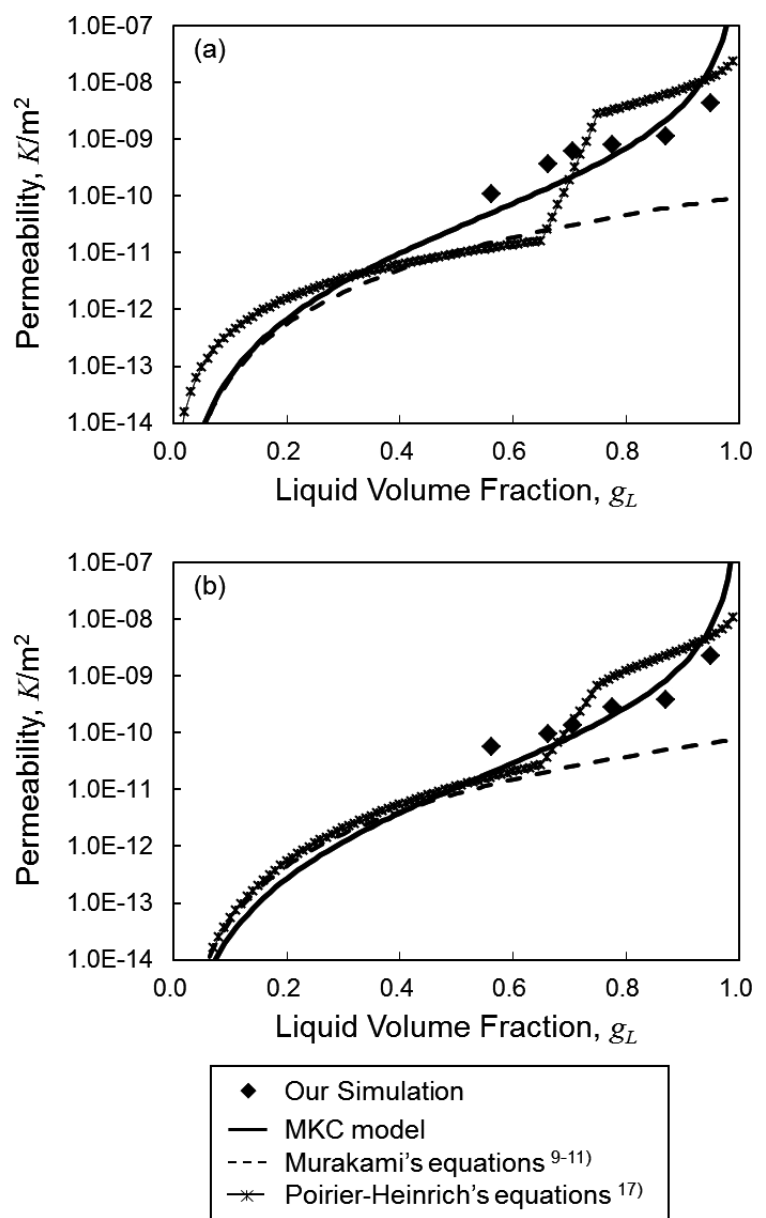

Fig. 8. Relationship between the liquid volume fractions and the permeability obtained using the empirical equations ${ }^{9-11,17)}$ and the MKC model for a PDAS of $320 \mu \mathrm{m}$ and an SDAS of $90 \mu \mathrm{m}$. (a) For a flow parallel to the primary arms. (b) For a flow normal to the primary arms. 

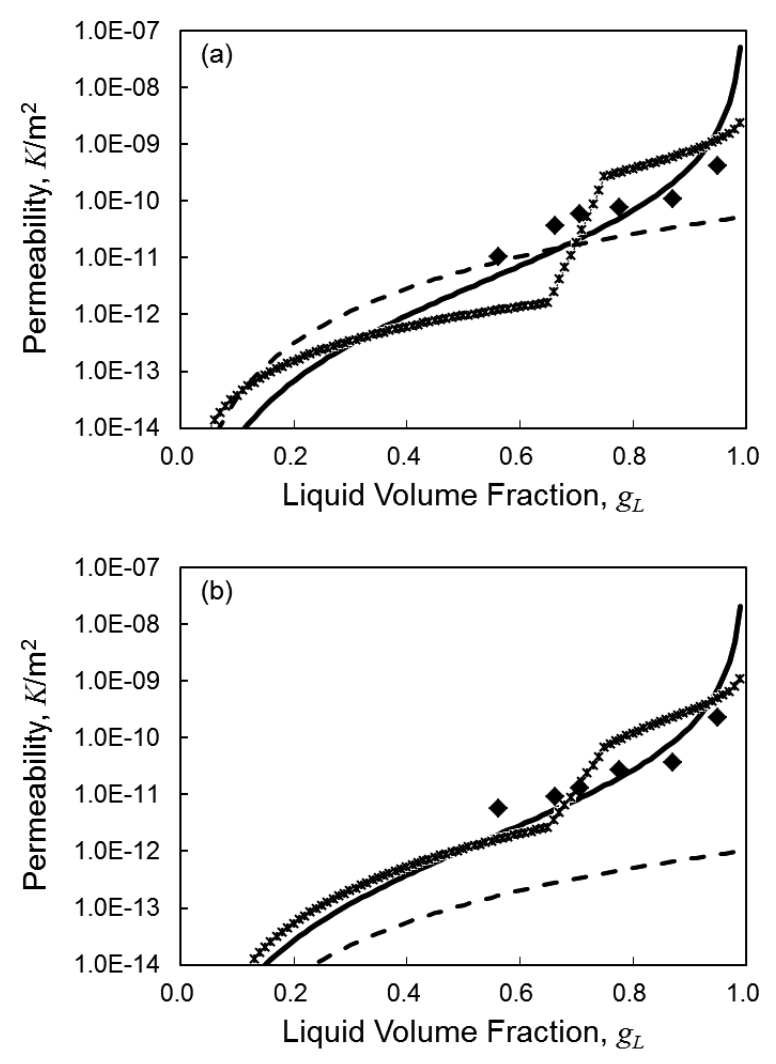

\begin{tabular}{ll|} 
- Our Simulation \\
- MKC model \\
-- Murakami's equations ${ }^{9-11)}$ \\
$*$ Poirier-Heinrich's equations ${ }^{17)}$
\end{tabular}

Fig. 9. Relationship between the liquid volume fractions and the permeability obtained using the empirical equations ${ }^{9-11,17)}$ and the MKC model for a PDAS of $100 \mu \mathrm{m}$ and an SDAS of $28 \mu \mathrm{m}$. (a) For a flow parallel to the primary arms. (b) For a flow normal to the primary arms.

Figure 8 shows the relationship between the liquid volume fractions and the permeability for a PDAS of 320 $\mu \mathrm{m}$ and an SDAS of $90 \mu \mathrm{m}$. In the same way, the values of the PDAS and SDAS are as determined in the experiments of Murakami et al., ${ }^{9-11)}$ and the value of $1 / S_{V}$ used is $1.65 \times$ $10^{-5} \mathrm{~m}$. For comparison, the results of our simulation are also shown in these figures. In both cases, for flows parallel to (Fig. 8(a)) and normal to (Fig. 8(b)) the primary arms, the relationship between the MKC model and both empirical equations was similar to that seen for the PDAS of $420 \mu \mathrm{m}$ and SDAS of $115 \mu \mathrm{m}$.

Figure 9 shows the relationship between the liquid volume fraction and the permeability for a PDAS of $100 \mu \mathrm{m}$ and an SDAS of $28 \mu \mathrm{m}$. In this case, the values of the PDAS and SDAS are original values obtained in the present work, but the ratio $d_{2} / d_{1}$ is almost the same as determined from the experiments of Murakami et al. ${ }^{9-11)}$ The value of $1 / S_{V}$ is 5.15 $\times 10^{-6} \mathrm{~m}$. For comparison, the results of our simulation are also shown in these figures. First, in the case of the flow parallel to the primary arms (Fig. 9(a)), the permeability determined from the MKC model was in good agreement with those obtained from both empirical equations. However, the curve determined from Murakami's equations crosses that from the MKC model at a liquid volume fraction of about 0.7 . This is observed only at a PDAS of $100 \mu \mathrm{m}$. Next, in the case of flow normal to the primary arms (Fig. 9(b)), the curve of permeability obtained using the MKC model is in fairly good agreement with that obtained using the PoirierHeinrich's equations. On the other hand, the curve from the Murakami's equations is much lower than the curves from the MKC model and the Poirier-Heinrich's equations. From these results, it can be concluded that it is difficult to evaluate quantitatively the permeability for different PDAS and SDAS using the Murakami's equations. On the other hand, the curve of permeability developed using the PoirierHeinrich's equations is artificial and unnatural.

In contrast, the MKC model can evaluate the permeability quantitatively under a wide range of conditions, and the curve obtained is natural. This further concludes that the MKC model is best to quantitatively determine the permeability for columnar dendritic structures.

\section{Quantitative Model of Permeability for Simulating Macrosegregation}

In the previous section, we concluded that the MKC model is a good quantitative model to determine permeability for columnar dendritic structures. In this section, we discuss a model of permeability for predicting macrosegregation by numerical simulation. In the numerical simulation of macrosegregation, it is important to predict exact positions or areas where macrosegregation occurs. To do this, it is necessary to evaluate exactly the permeability for dendritic structures, and as discussed, the MKC model is useful for this.

In order to use the $\mathrm{MKC}$ model in a numerical simulation, the values of $1 / S_{V}$ and $k_{m}$ for various dendritic structures must be continuously calculated during the simulation. The value of $k_{m}$, which is a function of the PDAS and the SDAS, is calculated relatively easily using a relationship between the dendrite arm spacing and the solidification conditions ${ }^{29)}$ (e.g. Eqs. (17) and (18)).

$$
\begin{aligned}
& d_{1}=4.3\left(\frac{D \Gamma \Delta T_{0}}{k_{0}}\right)^{1 / 4} V^{-1 / 4} G^{-1 / 2} \\
& d_{2}=5.5\left(M t_{f}\right)^{1 / 3}
\end{aligned}
$$

where $D$ is the diffusion coefficient in liquid, $\Gamma$ is the GibbsThomson coefficient, $\Delta T_{0}$ is the liquidus-solidus range at the initial alloy concentration, $k_{0}$ is the partition coefficient, $V$ is the velocity of interfacial movement, $G$ is the temperature gradient, $M$ is the materials parameter, and $t_{f}$ is the local solidification time. That is, $k_{m}$ can be evaluated by the physical properties of the alloy and the solidification conditions such as $G$ and $V$. These can be obtained during simulation. However, it is difficult to calculate the value of $1 / S_{V}$ during simulation because there is no information about microscopic dendritic structures in a macroscopic simulation. As a solution, we considered the use of an assumption that define $1 / S_{V}$ as a function of a characteristic length of dendrite such as $d_{1}, d_{2}$, and tip radius $r$. The function is given as follows:

$$
1 / S_{V}=f\left(d_{1}, d_{2}, r, \cdots\right)
$$

In the present work, for simplicity, we used the assumption that $1 / S_{V}$ is directly proportional to $d_{2}$. Figure 10 shows the relationship between the values of $1 / S_{V}$ and $d_{2}$ obtained from 


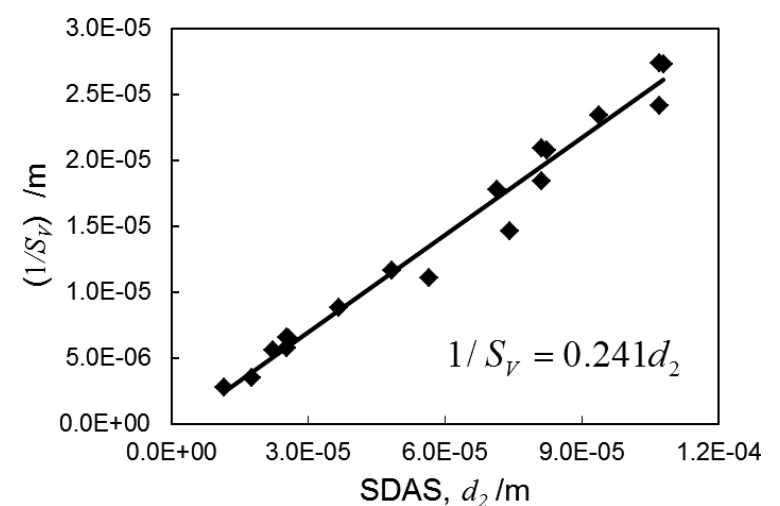

Fig. 10. Relationship between the SDAS and the inverse of surface area per volume for the three-dimensional columnar dendritic structures used in our flow calculations.
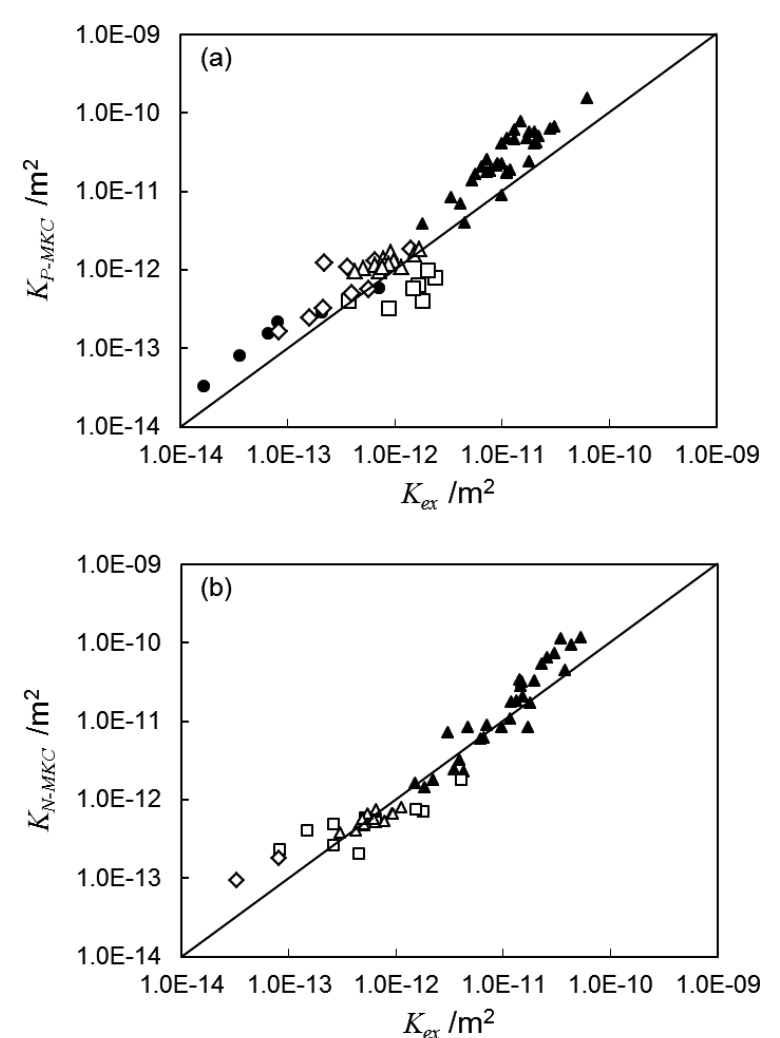

$\Delta$ Murakami et al. ${ }^{9,10)}$
$\Delta$ Liu et al. ${ }^{11)}$
$\bullet$ Streat and Weinberg ${ }^{3)}$
$\square$ Poirier et al..$^{13)}$
$\diamond$ Nasser-Rafi et al. ${ }^{12)}$

Fig. 11. Comparison of the permeability obtained from experiments and the MKC model. (a) For a flow parallel to the primary arms. (b) For a flow normal to the primary arms.

the three-dimensional columnar dendritic structures used in our simulation. From this relationship, $1 / S_{V}$ and $d_{2}$ are proportional to each other, and the constant of proportionality was about 0.241 . This is described as follows:

$$
1 / S_{V}=0.241 d_{2}
$$

From Eqs. (14), (15) and (20), the permeability for a flow parallel to the primary arms, $K_{P-M K C}$, becomes as follows:

$$
K_{P-M K C}=0.0194\left[\frac{d_{2}}{1+\left(d_{2} / d_{1}\right)}\right]^{2} \frac{g_{L}^{3}}{\left(1-g_{L}\right)^{2}} \ldots \ldots
$$

In addition, the permeability for a flow normal to the primary dendrites, $K_{N-M K C}$, becomes as follows:

$$
K_{N-M K C}=0.0097\left[\frac{d_{2}}{1+\left(d_{2} / d_{1}\right)+2\left(d_{2} / d_{1}\right)^{2}}\right]^{2} \frac{g_{L}^{3}}{\left(1-g_{L}\right)^{2}}
$$

These equations mean that the permeability for columnar dendritic structures can be determined from only the dendrite arm spacing and the liquid volume fraction. To examine the validity of these equations, we compared them with an experimental permeability, $K_{e x}$, where $d_{1}, d_{2}$, and $g_{L}$ were measured. ${ }^{9-14)}$ Figure 11 shows the comparison of $K_{P-M K C}$ and $K_{N-M K C}$ with $K_{e x}$ for flows parallel to and normal to the primary arms. Substituting the values of $d_{1}, d_{2}$, and $g_{L}$ measured experimentally into Eqs. (21) and (22), the values of $K_{P-M K C}$ and $K_{N-M K C}$ were calculated and compared with the values of $K_{e x}$ measured experimentally. For flows both parallel to and normal to the primary arms, the values of $K_{P \text { - }}$ $M K C$ and $K_{N-M K C}$ were in fairly good agreement with those of $K_{e x}$. Therefore, we confirmed that the MKC model employing the assumption of Eq. (20) is able to determine quantitatively the permeability for columnar dendritic structures and thus can be used for the numerical simulation of macrosegregation.

\section{Conclusion}

A new model (MKC model) that is able to quantitatively evaluate the permeability for columnar dendritic structures was developed by modifying the Kozeny constant in KozenyCarman's equation. The permeability calculated by the MKC model was in fairly good agreement with that obtained from simulations in our previous work and that measured experimentally by Murakami et al. ${ }^{9-11)}$ From these results, we confirmed that the MKC model was a valid method to quantitatively determine the permeability for columnar dendritic structures. Next, the permeability obtained using the MKC model was compared with those obtained using Murakami's equations ${ }^{9-11)}$ and the PoirierHeinrich's equations. ${ }^{17)}$ Results from Murakami's equations were in good agreement with those from the MKC model for low liquid volume fractions (less than about 0.6, the range of volume fractions used by Murakami et al.). ${ }^{9-11)}$ Moreover, in the case with a PDAS of $100 \mu \mathrm{m}$, the permeability determined for a flow normal to the primary arms using Murakami's equations was not in complete agreement with that obtained using the MKC model and the PoirierHeinrich's equations. The results obtained using PoirierHeinrich's equation was in good agreement with those obtained using MKC model, but the permeability varied artificially in the former case. From these results, we concluded that the MKC model performed best to quantitatively evaluate the permeability for columnar dendritic structures.

Moreover, we discussed the use of the quantitative model in the simulation of macrosegregation. On assuming $1 / S_{V}$ to be a function of $d_{2}$, the MKC model was demonstrated to determine the permeability from only the dendrite arm spacing and the liquid volume fraction. The permeability calculated using the MKC model with the above assumption was in fairly good agreement with that measured experimentally 
by several other researchers. From these results, we concluded that the MKC model is very effective to quantitatively evaluate the permeability for columnar dendritic structures.

\section{REFERENCES}

1) T. S. Piwonka and M. C. Flemings: Trans. Metall. Soc. AIME, 236 (1966), 1157

2) D. Apelian, M. C. Flemings and R. Mehrabian: Metall. Trans., 5 (1974), 2533

3) N. Streat and F. Weinberg: Metall. Trans. B, 7B (1976), 417.

4) D. R. Poirier and P. Ocansey: Mater. Sci. Eng., A171 (1993), 231.

5) D. R. Poirier and S. Ganesan: Mater. Sci. Eng., A157 (1992), 113.

6) T. Takahashi and I. Hagiwara: J. Jpn. Inst. Met., 29 (1965), 1152.

7) T. Takahashi, M. Kudoh and K. Yodoshi: J. Jpn Inst. Met., 43 (1975), 1086.

8) T. Takahashi, M. Kudoh and S. Nagai: Tetsu-to-Hagané, 68 (1982), 623.

9) K. Murakami, A. Shiraishi and T. Okamoto: Acta Metall., 31 (1983), 1417

10) K. Murakami, A. Shiraishi and T. Okamoto: Acta Metall., 32 (1984), 1423.

11) C. Y. Liu, K. Murakami and T. Okamoto: Mater. Sci. Technol., 5 (1989), 1148

12) R. Nasser-Rafi, R. Deshmukh and D. R. Poirier: Metall. Trans., 16A
(1985), 2263.

13) D. R. Poirier: Metall. Trans., 18B (1987), 245

14) K. Murakami and T. Okamoto: Acta Metall., 32 (1984), 1741.

15) S. Ganesan, C. L. Chan and D. R. Poirier: Mater. Sci. Eng., A151 (1992), 92.

16) M. S. Bhat, D. R. Poirier and J. C. Heinrich: Metall. Trans., 26B (1995), 1049.

17) D. R. Poirier and J. C. Heinrich: Mater. Charact., 32 (1994), 287.

18) J. C. Heinrich, D. R. Poirier and D. Nagelhout: Comput. Methods Appl. Mech. Eng., 133 (1996), 79.

19) J. C. Heinrich and D. R. Poirier: C. R. Mecanique, 332 (2004), 429.

20) S. G. R. Brown, J. A. Spittle, D. J. Jarvis and R. Walden-Bevan: Acta Mater., 50 (2002), 1559.

21) D. Bernard, O. Nielsen, L. Salvo and P. Cloetens: Mater. Sci. Eng., A392 (2005), 112.

22) D. Fuloria, P. D. Lee and D. Bernard: Mater. Sci. Eng., A494 (2008),

23) J. Madison, J. Spowart, D. Rowenhorst, L. K. Aagesen, K. Thornton and T. M. Pollock: Acta Mater., 58 (2010), 2864.

24) Y. Natsume, D. Takahashi, K. Kawashima, E. Tanigawa and K. Ohsasa: Tetsu-to-Hagané, 99 (2013), 117.

$25)$ P. C. Carman: Trans. Inst. Chem. Eng. (London), 15 (1937), 150

26) J. L. Fowler and K. L. Hertel: J. Appl. Phys., 11 (1940), 496.

27) R. R. Sullivan and K. L. Hertel: J. Appl. Phys., 11 (1940), 761

28) R. G. Santos and M. L. N. M. Melo: Mater. Sci. Eng., A391 (2005), 151

29) W. Kurz and D. J. Fisher: Fundamentals of Solidification, 4th ed., Trans Tech Publications Ltd, Switzerland, (1998), 83. 